\title{
'||||||||||||||||||||||||||||||||||||||||||||||||||||||||||||||||||.
}

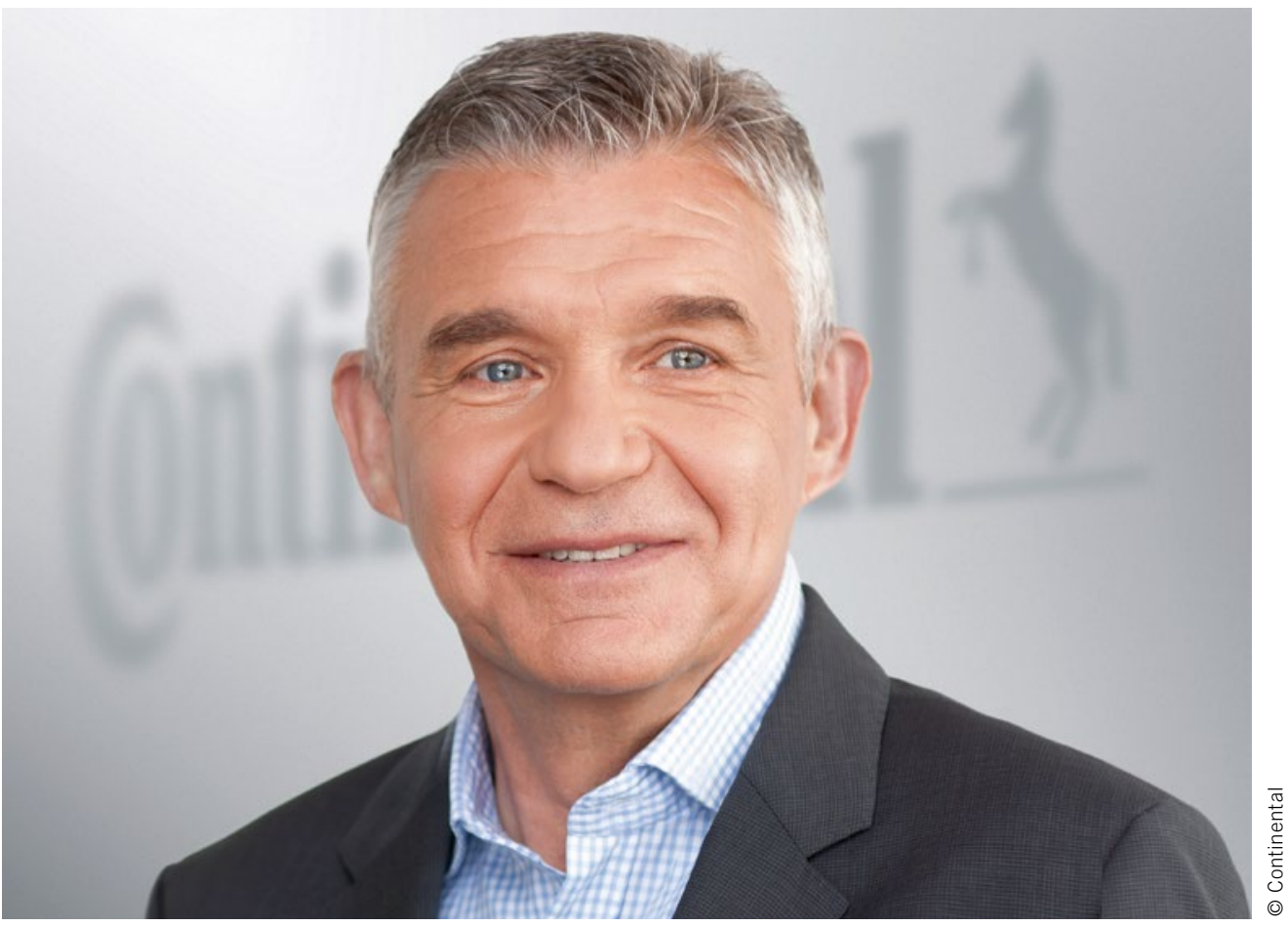

Dipl.-Ing. Karl Haupt

Executive Vice President Business Unit Advanced Driver Assistance Systems, Member of the Management Board of the Chassis \& Safety Division, Continental

\section{Sensory Impressions in Abundance}

The greatest risk of accidents in road traffic comes from the human. More than $90 \%$ of traffic accidents are due to some human error. The more we relieve and support drivers with technologies in the future, the more we will minimise the risk of accidents. Vehicles that can make the right decision within fractions of a second will reduce the rate of accidents considerably. Sophisticated driver assistance functions and automated driving are essential building blocks for relevant progress.

To allow automated vehicles to assume control from drivers, the vehicle has not only to continuously acquire, process and interpret data, but also build up ongoing contextual knowledge. The aim is that the car will be able to handle similar situations by itself with the help of examples it has learned. These examples are nothing other than human intuition. The vehicle needs to adapt human abilities. A basic requirement is therefore the comprehensive awareness and precise evaluation of the vehicle's surroundings, in the form of a highly reliable $360^{\circ}$ environment model.

The artificial brain of a vehicle - a central control unit - and its senses, that is to say, surrounding sensors such as cameras, radars and lidars, take on a key role for the environment model. They need to tackle different sensory impressions within split seconds: motorcycles and bicycles that weave past other vehicles, jaywalking pedestrians and suddenly merging traffic.
Added to this, there are many other situations of varying complexity. For this purpose, vast amounts of data must be processed and interpreted, and sensible actions derived from them. The difficulty here is that the error tolerance for the decisions an automated vehicle makes must match or even exceed that of a person.

This makes the task highly complex and challenging. At present, the big challenge is still the artificial brain. For automated driving, we need high-performance software and algorithms, plus considerably more sensors than in today's vehicles, as well as highly accurate maps. Only in this way can a precise and real-time image of the vehicle's surroundings be created and 3-D images of all static and dynamic objects constructed. However, more complexity and more information to process also means that more computing power is needed for analysis, comprehension, learning and training in contextual knowledge.

The development of control units and surrounding sensors is advancing just as rapidly as the gradually improving understanding of the vehicle's surroundings. This means that the day is approaching when vehicles will have an equal or even better understanding of its surroundings than persons do when driving. This understanding is therefore the key to automated driving. 